\title{
Central venous access devices in pediatric malignancies: a position paper of Italian Association of Pediatric Hematology and Oncology
}

\author{
Alessandro Crocoli ${ }^{1}$, Assunta Tornesello ${ }^{2}$, Mauro Pittiruti ${ }^{3}$, Angelica Barone ${ }^{4}$, Paola Muggeo ${ }^{5}$, Alessandro Inserra ${ }^{1}$, \\ Angelo Claudio Molinari ${ }^{6}$, Valeria Grillenzoni ${ }^{7}$, Viviana Durante ${ }^{8}$, Maria Pia Cicalese ${ }^{9}$, Giulio Andrea Zanazzo ${ }^{10}$, Simone Cesaro ${ }^{7}$ \\ 1 General and Thoracic Surgery Unit, Department of Surgery, Bambino Gesù Children's Hospital, IRCCS, Rome - Italy \\ 2 Pediatric Oncology Unit, Vito Fazzi Hospital, Lecce - Italy \\ 3 Department of Surgery, "Agostino Gemelli" Catholic University, Rome - Italy \\ 4 Pediatric Hematology and Oncology Unit, Department of Pediatrics, Azienda Ospedaliero-Universitaria di Parma, Parma - Italy \\ 5 Pediatric Hematology and Oncology, Pediatric Clinic, Azienda Ospedaliero-Universitaria Policlinico, Bari - Italy \\ 6 Thrombosis and Hemostasis Unit, Giannina Gaslini's Children Hospital, Genova - Italy \\ Pediatric Hematology Oncology, Azienda Ospedaliera Universitaria Integrata, Verona - Italy \\ 8 Pediatric Surgery Unit, University Policlinic Hospital, Modena - Italy \\ 9 Pediatric Immunology and Bone Marrow Transplantation Unit, San Raffaele Telethon Institute for Gene Therapy (HSR-TIGET), "San Raffa- \\ ele" Scientific Institute, Milan - Italy \\ 10 Institute for Maternal and Child Health, IRCCS "Burlo Garofolo", Trieste - Italy
}

\begin{abstract}
Introduction: Treatment of pediatric malignancies is becoming progressively more complex, implying the adoption of multimodal therapies. A reliable, long-lasting venous access represents one of the critical requirements for the success of those treatments. Recent technical innovations-such as minimally invasive procedures for placement, new devices and novel materials-have rapidly spread for clinical use in adult patients, but are still not consistently used in the pediatric population.

Methods: The Supportive Therapy Working Group of Italian Association of Hematology and Oncology (AIEOP) reviewed medical literature focusing on new aspects of central venous access devices (VADs) in pediatric patients affected by oncohematological diseases.

Results: Appropriate recommendations for clinical use in these patients have been discussed and formulated. Conclusions: The importance of the correct choice, management and use of VADs in pediatric oncohematological patients is a necessary prerequisite for an adequate standard of care, also considering the increased chances of cure and the longer life expectancy of those patients with modern therapies.
\end{abstract}

Keywords: Central venous catheter, Hematology, Oncology, Pediatric malignancies, PICC, Port

\section{Introduction}

In recent decades, the increasing use of venous access devices (VADs), either long term (LTVA) or medium term (MTVA), has provided a significant improvement in the treatment of children affected by oncohematologic diseases. According to the currently accepted terminology (1), LTVA is defined as

Accepted: August 26, 2014

Published online: October 14, 2014

Corresponding author:

Alessandro Crocoli, MD

General and Thoracic Surgery Unit

Department of Surgery and Centro Pediatrico

Bambino Gesù Basilicata

Bambino Gesù Children's Hospital

IRCCS, Rome, Italy

alessandro.crocoli@opbg.net a VAD with technical features which increase its stability in time, with an expected duration in the range of months and years: this category includes tunneled cuffed catheters and totally implantable venous ports. MTVA are VADs appropriate for a prolonged but not unlimited time (weeks or months) and for a discontinuous use (1): they include tunneled noncuffed central catheters, peripherally inserted central catheters (PICCS) and midline catheters.

A reliable and long-lasting central VAD permits a safer and easier administration of chemotherapy, supportive drugs, hyperhydration and hypertonic solutions, such as total parenteral nutrition, than via the peripheral vein.

Placement of a central VAD can be performed by surgeons, anesthesiologists, intensivists, interventional radiologists, oncologists or even nurses, depending on the type of device and the choice of venous access. LTVA and MTVA can be placed with different techniques (venous cutdown, "blind" percutaneous venipuncture guided by anatomical 
landmarks, ultrasound-guided percutaneous puncture and cannulation of the vein). Placement of a central line always carries potential risks for the patient, with relevant differences in the type and severity of complication, depending on which vein, which technique and which device have been chosen.

The objective of this position paper, promoted by the Italian Association of Pediatric Oncology and Hematology (AIEOP) and developed by the Supportive Therapy Working Group (ST-WG) of AIEOP, is to provide practical recommendations for indication, choice, placement and removal of LTVA and MTVA in pediatric oncohematologic patients. The present document also includes recommendations on the prevention of early and late noninfective complications potentially related to VAD insertion.

\section{Methods}

The ST-WG developed this position paper in collaboration with the AIEOP working groups on Infection, Coagulation, Surgery and Nursing. A joint committee was established, including the ST-WG and one to two members from each of the other working groups. During a preliminary meeting, the topics and the literature search were defined. In the National Library of Medicine's MEDLINE database, we searched all clinical papers (clinical trials, retrospective studies, case reports, reviews and consensus papers) dealing with central venous access in pediatric cancer patients; only papers published between January 2001 and January 2014, in English language and available as full text, were considered. A first selection was performed by the ST-WG, excluding papers of poor relevance or not focused on MTVAs or LTVAs. All papers in full text were examined by each member of the joint committee. During a second meeting, the committee discussed the clinical evidence of the most relevant papers in each area (indication/choice of the device; choice of access/insertion technique; prevention of mechanical complications; removal of the device) and specific recommendations were suggested. The ST-WG prepared a final document which was approved by the joint committee in a third meeting.

The main recommendations are summarized in Table I and discussed below.

\section{Results and Discussion}

\section{Indications for the Positioning of LTVA and MTVA; Selection of the Device}

The choice of the VAD should be based on the patient's needs, taking into account duration and type of oncology/ hematology treatment. There is general consensus that any chemotherapy treatment in pediatric patients should be delivered via an appropriate central venous access, as the risks associated with the infusion of vesicant or irritant drugs via a peripheral venous access are not acceptable. Many supportive therapies adopted in pediatric cancer patients, such as parenteral nutrition, also demand for a central line: most current guidelines state that peripheral lines (short and long i.v. cannulas; midline catheters) are inappropriate
TABLE I - SUmmary of AIEOP recommendations

\section{Indications and selection criteria}

For long-term treatments, ports should be preferred for intermittent use and cuffed tunneled catheters for frequent/ continuous use.

For short- to medium-term treatments, PICCs are a valid option, but they should be inserted only when deep veins of the arm are of appropriate diameter.

There is no evident advantage of silicon vs. polyurethane.

Double-lumen VADs should be used only in selected cases.

Catheters inserted in the femoral vein for medium- to long-term treatments should be tunneled away from the groin.

Prevention of early complications

Ultrasound should be used for diagnosis of local pathologic conditions before the procedure and for diagnosis of procedure-related complications.

Ultrasound is necessary to assess vein patency before the procedure and to choose the vein most appropriate in terms of caliber, depth and position.

The internal diameter of the vein to be cannulated-as assessed by ultrasound-should be at least three times the external diameter of the catheter, so as to reduce the risk of venous thrombosis.

Ultrasound-guided venipuncture should be adopted, so as to reduce the risk of early and late complications.

Venous cannulation by surgical cutdown should be avoided.

"Blind" percutaneous puncture of central veins ("landmark" venipuncture) should be avoided.

In newborn and infants, internal jugular vein and brachio-cephalic vein are usually the first options for ultrasound-guided central venous access.

Soon after procedures potentially associated with pleural damage, pneumothorax should be excluded by ultrasound scan of the intercostal space.

A specifically and properly trained vascular team should be implemented, so to reduce both insertion-related and managementrelated complications.

\section{Prevention of late mechanical complications}

Noncuffed catheters should be permanently secured, preferably by a sutureless device.

Cuffed catheters should be secured for at least 2-4 weeks, preferably by a sutureless device.

Valved and nonvalved catheters have the same expected incidence of complications.

\section{Removal}

Local anesthesia/sedation is used to remove a cuffed tunneled device whereas general anesthesia is usually needed to remove a port.

After removing the device, skin should be closed with absorbable sutures and/or glue and an occlusive dressing should be applied for at least 48 hours.

Complete removal of the cuff is recommended.

Embolized fragments of the catheter should be removed by nonsurgical endovascular procedure.

In case of device removal because of infection, a new device should be inserted at different times and using a different venous approach.

AIEOP = Italian Association of Pediatric Oncology and Hematology; PICC = peripherally inserted central catheter; $V A D=$ vascular access device 
for delivering solutions with high osmolarity, or $\mathrm{pH}>9$ or $<5$ (2). Short-term central venous access - such as nontunneled centrally inserted central venous catheters (CVCs)-are appropriate for intrahospital use and/or for a short period of time, but not as a long-standing venous access (1). Most children with malignancies require a reliable, stable venous access for discontinuous, extrahospital use: this is usually achieved by MTVA or LTVA, depending on the expected duration of need for the access.

Noncuffed PICCs are commonly considered as MTVA (1, 3); they may be a valid option for short- and medium-term durations of treatment, particularly when general anesthesia is contraindicated, since they can be inserted at bedside under local anesthesia and/or mild sedation (4-7). Though currently considered appropriate particularly for medium-term venous access (3), there is a growing evidence-particularly in adult cancer patients - that they might be used for a long period of time $(8,9)$.

Considering LTVA, there is no striking difference in terms of general clinical performance between tunneled cuffed catheters (such as Broviac, Hickman, Leonard, cuffed Groshong, ProLine) and totally implanted venous ports, although in children who require frequent venous access for blood sampling, parenteral nutrition and complex intravenous therapies, such as those eligible for high-dose chemotherapy and hematopoietic stem cell transplant, tunneled cuffed catheters are usually preferred. On the other hand, children requiring less intensive support and undergoing chemotherapy schedules with a 3-6 week interval, or adolescents, who may not accept an external device, are eligible for a port. In other words, venous ports are recommended for prolonged but intermittent vascular access while tunneled cuffed catheters are recommended for prolonged but frequent/continuous vascular access (10-12). Cuffed PICCs are now available and may have a future role as LTVAs, as an alternative option to centrally inserted tunneled cuffed catheters.

\section{Choice of material}

MTVAs and LTVAs are made of catheters of different materials, either silicon or polyurethane. There is no evidence of any difference between silicon and polyurethane in terms of risk of infective and thrombotic complications in the adult or in the pediatric population $(13,14)$. Silicon catheters have traditionally been the first choice for LTVA in pediatric patients (11, 15-18), although polyurethane catheters-and specially power injectable polyurethane catheters, which are made of third-generation polyurethanes-are as biocompatible as silicone catheters but less fragile; also, they are compatible with higher flow rates and are ideal for injection of contrast medium (19).

Table II summarizes the main types of external long-term and medium-term CVCs.

\section{Caliber of catheter}

The outer caliber of the catheter should be decided on the basis of the inner diameter of the vein, so as to prevent venous thrombosis (20). The caliber of vein should be measured by ultrasound scan (21). Ideally, the outer diameter of
TABLE II - Central venous catheters commonly used in the pediatric population

\begin{tabular}{|c|c|c|c|c|}
\hline \multicolumn{5}{|c|}{ Long term (tunneled, cuffed external VADs) } \\
\hline & Material & Tip & Lumen & French \\
\hline Broviac & Silicon & No valve & 1 & $\begin{array}{c}2.7,4.2,6.6 \\
7,9.6\end{array}$ \\
\hline Hickman & Silicon & No valve & 2 & 7,12 \\
\hline Leonard & Silicon & No valve & 2 & 10 \\
\hline Groshong & Silicon & Distal valve & 1 or 2 & $5.5,78,9$ \\
\hline ProLine & P.I. PUR & No valve & 1 or 2 & 5,6 \\
\hline \multicolumn{5}{|c|}{ Medium term (PICCs) } \\
\hline Silicon PICC & Silicon & No valve & 1 or 2 & $3,4,5$ \\
\hline $\begin{array}{l}\text { Groshong } \\
\text { PICC }\end{array}$ & Silicon & Distal valve & 1 or 2 & 4,5 \\
\hline $\begin{array}{l}\text { Solo, PASV } \\
\text { PICC }\end{array}$ & P.I. PUR & $\begin{array}{l}\text { Proximal } \\
\text { valve }\end{array}$ & 1 or 2 or 3 & $3,4,5,6$ \\
\hline PASV PICC & PUR & $\begin{array}{l}\text { Proximal } \\
\text { valve }\end{array}$ & 1 or 2 & 4,5 \\
\hline PUR PICC & PUR & No valve & 1 or 2 & 4,5 \\
\hline P.I. PUR PICC & P.I. PUR & No valve & 1 or 2 or 3 & $3,4,5,6$ \\
\hline
\end{tabular}

PASV = pressure-activated safety valve; P.I. = power injectable; PICC = peripherally inserted central catheter; PUR = polyurethane; VAD = vascular access device.

the catheter should be equal or smaller than one third of the internal diameter of the vein: for example, a $3 \mathrm{Fr}(=1 \mathrm{~mm})$ catheter is appropriate for a vein whose diameter is $9 \mathrm{Fr}$ (3 $\mathrm{mm})$ or larger $(13,16,22,23)$.

Double-lumen catheters, either LTVA or MTVA, are indicated in patients undergoing hematopoietic stem cell transplantation, in critically ill patients and in any patient needing chronic infusion of noncompatible solutions, such as the case of pediatric patients candidate to both parenteral nutrition and chemotherapy $(12,24,25)$, although some data suggest that the use of double-lumen devices might be associated with a higher risk of infection compared to single-lumen devices (18).

\section{Choice of the vein}

Central lines are characterized by the location of the tip of the catheter close to the cavo-atrial junction, either in the lower third of the superior vena cava or in the upper part of the atrium: this can be achieved by cannulation of either central or peripheral veins. For centrally inserted VADs, veins on the right side are preferred, so that the catheter may follow a direct path toward the atrio-caval junction. Data in adult patients suggest that the insertion on the right side may be associated with a lower incidence of thrombosis (26), although pediatric data in this regard are not available. In the preultrasound era, the internal jugular vein was considered the first choice, in terms of anatomical location and accessibility, while the "blind" puncture and cannulation of the subclavian vein was considered to be 
more dangerous, especially if the patient was a newborn or infant. In the ultrasound era, many different options for central venous cannulation are now available (internal jugular, external jugular, brachio-cephalic, subclavian and axillary veins) (21). The most appropriate vein should be chosen after proper ultrasound examination, considering the caliber of the vein and the potential risk associated with its cannulation $(13,21)$.

PICCs can be placed in any deep vein of the arm between the elbow and axilla (brachial vein, basilic vein, axillary vein and even cephalic vein in selected cases), but since the minimum size of the PICC is $3 \mathrm{Fr}$ there is a limited indication in newborns and infants due to the smaller venous caliber (13, 19). There is no clear recommendation as regards choosing left vs. right side, while the deep veins of the upper arm (brachial and basilic veins, which can be cannulated by ultrasound guidance) should be preferred over the superficial veins of the antecubital fossa (2). Side, vein and puncture site should be decided after proper ultrasound scan of the vasculature of the arm (21). The presence of veins of insufficient diameter (such as $<3 \mathrm{~mm}$ or $<4 \mathrm{~mm}$ when, respectively, a $3 \mathrm{Fr}$ or $4 \mathrm{Fr}$ catheter is required) is a contraindication to PICC insertion.

LTVA (tunneled cuffed catheters and ports) as well as MTVA (tunneled noncuffed long catheters) are sometimes also inserted by the femoral access, with the tip of the catheter in the upper portion of the inferior vena cava: this can be a suitable option in patients with superior vena cava obstruction syndrome. The most relevant complication of femoral access is infection: should this route be chosen for a LTVA or MTVA, tunneling has to be performed away from the groin, along the thigh with exit site on the side of the knee or upward to the periumbilical region, so as to reduce the risk of infection by germs originating from the groin $(7,13,23)$.

\section{Placement of LTVA and MTVA in pediatric patients}

Before the introduction of ultrasound guidance in clinical practice, placement of centrally inserted catheters by "blind" venipuncture or venous cutdown had been widely used in pediatric patients.

"Blind" puncture of central veins is associated with the risk of failure, repeated punctures, accidental arterial injury and pneumothorax due to accidental pleural damage. Accidental damage to the pleura is significantly more frequent after subclavian than after jugular venipuncture (27). A peculiar complication is the so-called pinch-off syndrome, a compression of the catheter between the first rib and the clavicle, resulting in malfunction, obstruction, fissure and/or fracture and embolization of a catheter fragment: this syndrome is exclusively associated with "blind" infraclavicular cannulation of the subclavian vein $(12,22,23)$.

The use of the surgical technique for the isolation and cannulation of the vein (venous cutdown) is associated with a relevant incidence of tissue trauma, failure and local complications even in experienced hands. Vessel thrombosis occurs more frequently, because of the dissection of tissues and the direct section and ligation of the vessel. Also, the surgical isolation of the vein entails a higher risk of infection at the site of entry of the catheter $(2,28,29)$.
In adult patients, the use of ultrasound guidance to select and puncture the vein is clearly associated with a significantly higher rate of success and a significantly lower incidence of mechanical, infective and thrombotic complications if compared to surgical "cutdown" or "blind percutaneous techniques" (21). Ultrasound permits visibility of the progression of the needle in the target vessel, reducing the likelihood of accidental arterial puncture or accidental damage to nerves and other surrounding structures (21).

Though the evidence in pediatric patients is still limited, most studies suggest that ultrasound guidance should become the state of the art of venipuncture in children and neonates, as it is already in adults $(13,30-32)$. The most important advantage of ultrasound is to allow the choice of the most appropriate vein after a scan of all possible options (21). By ultrasound guidance, puncture and cannulation of the vein are quicker and easier, as well as associated with fewer complications; moreover, the reduced invasiveness of the procedure consistently reduces the risk of infection at the insertion site and the risk of catheter-related thrombosis $(13,30,33-36)$.

Also, ultrasound permits real-time diagnosis of preexisting vascular anomalies (malformations, anatomic variants or thrombosis) and of early complications (hematoma postinjection, extravasation, dissection of the vessel or stenosis, pneumothorax, etc.) $(12,37)$.

In patients with a previous placement of a venous access or other risk factors for venous thrombosis (surgery of the neck or superior vena cava syndrome), ultrasound allows the assessment of the patency of the vessel to be cannulated $(12,21)$.

The central veins in adults and children that can be cannulated under ultrasound guidance are the internal jugular vein, the external jugular vein (in its deeper path), the brachialcephalic, subclavian (usually by a supraclavicular approach), the axillary vein (by infraclavicular approach) and the cephalic vein (in the infraclavicular region). In newborns and infants, the only veins of significant size apt to be cannulated are usually the internal jugular and brachio-cephalic $(13,31)$.

After the ultrasound-guided puncture of the vessel, a guidewire is inserted and directed to the cavo-atrial junction. In neonates and infants, ultrasound is also useful to guide the wire into the superior vena cava toward the right atrium; the final position of the tip can be verified by echocardiography. The guidewire should never be inserted to a length superior than the distance between puncture site and right atrium, since even the softest guidewire may damage the heart wall or provoke dangerous arrhythmias. A guidewire can be advanced to the inferior vena cava only if the maneuver is performed under fluoroscopic guidance, but this is not routinely recommended and it might be risky, especially in neonates and infants. After the insertion of the guidewire-as all MTVAs and LTVAs are currently inserted by the modified Seldinger technique-a peel-away introducer is advanced over the guidewire until the lower portion of the superior vena cava. The guidewire is removed and the catheter is threaded through the introducer.

The optimal position of the catheter tip is at the junction between the right atrium and the superior vena cava $(1,2)$. The tip location should be ideally verified in real time during the procedure (by fluoroscopy, by intracavitary electro- 
cardiogram (ECG) or by echocardiography) or-as a second option-soon after the procedure (by chest x-ray or by echocardiography). In the case of femoral access, the recommended level of the catheter tip is in the inferior vena cava below the renal veins. Before starting to use an LTVA or MTVA, the correct position of the tip must be verified and documented in the medical record $(2,21,33,34)$. Intracavitary ECG and/ or echocardiography is safer and more accurate than fluoroscopy for verifying tip location and is becoming increasingly used in clinical practice, although the experience in pediatric patients is still limited $(38,39)$.

In the case of subclavian vein access, ultrasound examination of the intercostal spaces is a suitable examination for the early diagnosis of a pneumothorax secondary to the procedure. Immediate ultrasound scan of the pleural space for detecting the "sliding sign" is needed in any difficult puncture of the subclavian or axillary vein, and particularly if the patient complains of shortness of breath, discomfort or pain worsening after catheter placement; chest $\mathbf{x}$-ray is less accurate and less immediately available in this regard $(21,40)$.

Proper training of the vascular team (41) and proper choice of methods and materials (13) are the key factors in reducing operative time and risk of intra- and postprocedural complications. When the same team is also responsible for the management of the venous access, late complications are also reduced (37).

\section{Prevention of late mechanical complications of CVC}

The more frequent late mechanical complications are dislocation of the catheter, migration of the tip, lumen occlusion and catheter rupture. All these complications are clinically associated with a malfunction of the device, often as a failure to draw from and/or to infuse through the device. Dislocation is the leading cause of premature loss of external venous access, especially in younger children (11). Dislocation can be minimal (less than 1-2 cm), partial (more than $2 \mathrm{~cm}$ ) or complete (complete removal of the device). All noncuffed central catheters-both tunneled and nontunneled-should be permanently secured to the skin by an adequate sutureless device (29), although securement by a sutureless device is also needed for cuffed tunneled catheters, at least in the first 3-4 weeks after placement. Management of the exit site should be performed by trained personnel and in accordance with the characteristics of the VAD and the type of dressing used. Sutures should be used only in very selected cases, that is, when attachment of the sutureless device to the skin does not appear to be reliable. A new securement device which is apparently more effective than sutures in preventing dislocation consists in a device which anchors the catheter directly to the subcutaneous tissue $(42,43)$, although clinical studies with this device in pediatric patients are not yet available.

The tunneled cuffed catheters have a Dacron cuff, which requires 2 to 4 weeks to ensure a proper securement to the subcutaneous tissue of the patient $(12,13,44)$. The cuff must be placed inside the tunnel at least $2 \mathrm{~cm}$ far from the exit site, as recommended by the manufacturer. A cuff inappropriately placed too close to the exit site is a well-recognized cause of dislocation of tunneled cuffed catheters (8).
Catheter fixation to the muscular fascia with nonabsorbable braided sutures has also been used for reducing the risk of dislocation in cases where a delay of the healing process and cuff anchoring is expected (prolonged use of corticosteroids, Omenn syndrome, epidermolysis bullosa) (13). The new sutureless device anchoring the catheter to the subcutaneous tissue $(42,43)$ will most likely make these surgical securement obsolete.

The reservoir of venous ports is sometimes secured to the underlying muscle fascia (10), although this is not considered to be mandatory in all cases (26).

The migration of the tip is another common mechanical complication that can occur even without external dislocation of the catheter and is more frequent with silicon catheters, due to the softness of the material. It is often associated with inappropriately "high" position of the tip (i.e., when the tip does not enter the lower third of the superior vena cava).

The occlusion of the lumen is more frequent with smaller caliber catheters and/or when proper protocols of flushing and locking are not adopted. Valved catheters are available for both MTVA and LTVA, although there is no evidence that valved catheters (either with distal or with proximal valves) may be associated with a lower risk of occlusion (or of any other complication) (45-48). Lumen occlusion is basically related to the local policies of flushing and locking the VAD, rather than to the technique of insertion $(2,15)$.

Mechanical lesions, breakage and fracture of the deviceparticularly of PICCs - are significantly more frequent with silicon than polyurethane catheters. The "pinch-off" syndrome has been a frequent cause of catheter malfunction and fracture in the past, although it is bound to disappear in the ultrasound era, as it was invariably associated with the "blind" puncture of the subclavian vein by infraclavicular approach.

\section{Removal of the CVC}

Removal of MTVA or LTVA must be performed by experienced personnel, in an appropriate setting. Noncuffed catheters such as PICCs may be easily removed at the patient's bedside, whereas the removal of an LTVA requires proper aseptic conditions (operating or procedure room). Before removing a tunneled cuffed catheter, the cuff should be located by palpation or by ultrasound. If the cuff is placed quite distant from the exit site, removal may require a new incision of the overlying skin $(49,50)$.

In pediatric patients, local anesthesia/sedation is used to remove a cuffed tunneled device whereas general anesthesia is usually needed to remove a port (49-51).

After removing the device, the exit site must be compressed for at least 5 minutes to reduce local bleeding. The exit point or points must be closed with absorbable sutures and/or glue; an occlusive dressing should be applied for at least 48 hours to avoid the risk of air embolism $(49,50)$.

The complete removal of the cuff is necessary, since retained cuffs or cuff fragments may become a source of infection, create false radiological images or cause unsatisfactory cosmetic results (49-51).

In the case of catheter embolization, the removal of the fragment usually requires a nonsurgical endovascular procedure by an interventional radiologist $(22,52)$. 
In case of loss of the catheter due to complete dislocation, the VAD can be repositioned in the same vein. In case of infection, the procedures of VAD removal and repositioning are carried out at different times to avoid the risk of infecting the new device, and preferentially using a different venous access (53).

\section{Conclusions}

As far as we know, this is the first position paper reviewing and discussing the issues of choice, placement and removal of LTVAs from the perspective of oncologic pediatric patients. Despite the referenced literature, there is far less evidence for children than for adults. The use of ultrasound guidance is an important innovation to reduce invasiveness and lower the incidence of both early and late complications. The investment needed for adequate staff training, and the acquisition of appropriate materials and appropriate methodologies is counterbalanced by an improvement in patient safety and a lower morbidity and mortality. In pediatric oncohematology patients these results are even more important in the light of an increased chance of cure and a longer life expectancy.

\section{Disclosures}

Financial support: None.

Conflict of interest: None.

\section{References}

1. Gallieni M, Pittiruti M, Biffi R. Vascular access in oncology patients. CA Cancer J Clin. 2008;58(6):323-346.

2. Pittiruti M, Hamilton H, Biffi R, MacFie J, Pertkiewicz M; ESPEN. ESPEN guidelines on parenteral nutrition: central venous catheters (access, care, diagnosis and therapy of complications). Clin Nutr. 2009;28(4):365-377.

3. Loveday HP, Wilson JA, Pratt RJ, et al; UK Department of Health. epic3: national evidence-based guidelines for preventing healthcare-associated infections in NHS hospitals in England. J Hosp Infect. 2014;86(Suppl 1):S1-S70.

4. Abedin S, Kapoor G. Peripherally inserted central venous catheters are a good option for prolonged venous access in children with cancer. Pediatr Blood Cancer. 2008;51(2):251-255.

5. Al Raiy B, Fakih MG, Bryan-Nomides N, et al. Peripherally inserted central venous catheters in the acute care setting: a safe alternative to high-risk short-term central venous catheters. Am J Infect Control. 2010;38(2):149-153.

6. Matsuzaki A, Suminoe A, Koga Y, Hatano M, Hattori S, Hara T. Long-term use of peripherally inserted central venous catheters for cancer chemotherapy in children. Support Care Cancer. 2006;14(2):153-160.

7. Silberzweig JE, Sacks D, Khorsandi AS, Bakal CW; Society of Interventional Radiology Technology Assessment Committee. Reporting standards for central venous access. J Vasc Interv Radiol. 2003;14(9 Pt 2):S443-S452.

8. Cotogni $P$, Pittiruti $M$, Barbero $C$, Monge $T$, Palmo A, Boggio Bertinet D. Catheter-related complications in cancer patients on home parenteral nutrition: a prospective study of over 51,000 catheter days. JPEN J Parenter Enteral Nutr. 2013;37(3): 375-383.

9. Cotogni P, Barbero C, Garrino C, et al. Peripherally inserted central catheters in non-hospitalized cancer patients: 5-year results of a prospective study. Support Care Cancer. 2014; Epub ahead of print.

10. Cil BE. Radiological placement of chest ports in pediatric oncology patients. Eur Radiol. 2004;14(11):2015-2019.

11. Cesaro S, Corrò R, Pelosin A, et al. A prospective survey on incidence and outcome of Broviac/Hickman catheter-related complications in pediatric patients affected by hematological and oncological diseases. Ann Hematol. 2004;83(3):183-188.

12. Marcy PY. Central venous access: techniques and indications in oncology. Eur Radiol. 2008;18(10):2333-2344.

13. Pittiruti M. Ultrasound guided central vascular access in neonates, infants and children. Curr Drug Targets. 2012;13(7): 961-969.

14. Cohen AB, Dagli M, Stavropoulos SW Jr, et al. Silicone and polyurethane tunneled infusion catheters: a comparison of durability and breakage rates. J Vasc Interv Radiol. 2011;22(5): 638-641.

15. Cesaro S, Tridello G, Cavaliere M, et al. Prospective, randomized trial of two different modalities of flushing central venous catheters in pediatric patients with cancer. J Clin Oncol. 2009; 27(12):2059-2065.

16. Farinasso L, Bertorello N, Garbarini L, et al. Risk factors of central venous lines-related thrombosis in children with acute lymphoblastic leukemia during induction therapy: a prospective study. Leukemia. 2007;21(3):552-556.

17. Fratino G, Molinari AC, Parodi S, et al. Central venous catheterrelated complications in children with oncological/hematological diseases: an observational study of 418 devices. Ann Oncol. 2005;16(4):648-654.

18. Pinon M, Bezzio S, Tovo PA, et al. A prospective 7-year survey on central venous catheter-related complications at a single pediatric hospital. Eur J Pediatr. 2009;168(12):1505-1512.

19. Pittiruti M, Brutti A, Celentano D, et al. Clinical experience with power-injectable PICCs in intensive care patients. Crit Care. 2012;16(1):R21.

20. Nifong TP, McDevitt TJ. The effect of catheter to vein ratio on blood flow rates in a simulated model of peripherally inserted central venous catheters. Chest. 2011;140(1):48-53.

21. Lamperti M, Bodenham AR, Pittiruti M, et al. International evidence-based recommendations on ultrasound-guided vascular access. Intensive Care Med. 2012;38(7):1105-1117.

22. Knutstad K, Hager B, Hauser M. Radiologic diagnosis and management of complications related to central venous access. Acta Radiol. 2003;44(5):508-516.

23. Skinner R, Koller K, Mclntosh N, McCarthy A, Pizer B; United Kingdom Children's Cancer Study Group (UKCCSG); Paediatric Oncology Nursing Forum (PONF) Supportive Care Group. Prevention and management of central venous catheter occlusion and thrombosis in children with cancer. Pediatr Blood Cancer. 2008;50(4):826-830.

24. Dezfulian C, Lavelle J, Nallamothu BK, Kaufman SR, Saint S. Rates of infection for single-lumen versus multilumen central venous catheters: a meta-analysis. Crit Care Med. 2003;31(9): 2385-2390.

25. Chernecky C, Macklin D, Nugent K, Waller JL. The need for shared decision-making in the selection of vascular access devices: an assessment of patients and clinicians. J Vasc Access. 2002; 7(3):34-40.

26. Pittiruti M, La Greca A, Emoli A, Scoppettuolo G. Il protocollo ISALT 2 per l'impianto degli accessi venosi centrali a lungo termine: una proposta GAVeCeLT per un approccio più sicuro e costo-efficace. Osp Ital Chir. 2010;16:359-368.

27. Ruesch S, Walder B, Tramèr MR. Complications of central venous catheters: internal jugular versus subclavian access-a systematic review. Crit Care Med. 2002;30(2):454-460. 
28. Avanzini S, Guida E, Conte M, et al. Shifting from open surgical cut down to ultrasound-guided percutaneous central venous catheterization in children: learning curve and related complications. Pediatr Surg Int. 2010;26(8):819-824.

29. O'Grady NP, Alexander M, Burns LA, et al.; Healthcare Infection Control Practices Advisory Committee (HICPAC). Guidelines for the prevention of intravascular catheter-related infections. Clin Infect Dis. 2011;52(9):e162-e193.

30. Bruzoni M, Slater BJ, Wall J, St Peter SD, Dutta S. A prospective randomized trial of ultrasound- vs landmark-guided central venous access in the pediatric population. J Am Coll Surg. 2013; 216(5):939-943.

31. Di Nardo M, Tomasello C, Pittiruti M, et al. Ultrasound-guided central venous cannulation in infants weighing less than 5 kilograms. J Vasc Access. 2011;12(4):321-324.

32. Verghese ST, McGill WA, Patel RI, Sell JE, Midgley FM, Ruttimann UE. Comparison of three techniques for internal jugular vein cannulation in infants. Paediatr Anaesth. 2000;10(5):505-511.

33. Augoustides JG, Horak J, Ochroch AE, et al. A randomized controlled clinical trial of real-time needle-guided ultrasound for internal jugular venous cannulation in a large university anesthesia department. J Cardiothorac Vasc Anesth. 2005; 19(3):310-315.

34. Grebenik CR, Boyce A, Sinclair ME, Evans RD, Mason DG, Martin B. NICE guidelines for central venous catheterization in children. Is the evidence base sufficient? Br J Anaesth. 2004; 92(6):827-830.

35. Campisi C, Biffi R, Pittiruti M. Catheter related central venous thrombosis: the development of a nationwide consensus paper in Italy. JAVA. 2007;12(1):38-46.

36. Debourdeau P, Farge D, Beckers $M$, et al. International clinical practice guidelines for the treatment and prophylaxis of thrombosis associated with central venous catheters in patients with cancer. J Thromb Haemost. 2013;11(1):71-80.

37. Hancock-Howard R, Connolly BL, McMahon M, et al. Costeffectiveness analysis of implantable venous access device insertion using interventional radiologic versus conventional operating room methods in pediatric patients with cancer. J Vasc Interv Radiol. 2010;21(5):677-684.

38. Pittiruti M, La Greca A, Scoppettuolo G. The electrocardiographic method for positioning the tip of central venous catheters. J Vasc Access. 2011;12(4):280-291.

39. Rossetti F, Pittiruti M, Lamperti M, Graziano U, Celentano D, Capozzoli G. The intracavitary ECG method for positioning the tip of central venous access in pediatric patients: results of an Italian multicenter study. J Vasc Access. 2012;13:357-365.

40. Barrett $M$, Imeson J, Leese $D$, et al. Factors influencing early failure of central venous catheters in children with cancer. J Pediatr Surg. 2004;10(10):1520-1523.
41. Lamperti M, Moureau N, Kelly LJ, et al. Competence in paediatric central venous lines placement. $\mathrm{Br} J$ Anaesth. 2014;112(2):383.

42. Elen Hughes M. Reducing PICC migrations and improving patient outcomes. Br J Nurs. 2014;23(Suppl 2):S12-S18, S14-S18.

43. Cordovani D, Cooper RM. A prospective trial on a new sutureless securement device for central venous catheters. Can J Anesth. 2013;60:504-505.

44. Fratino G, Avanzini S, Molinari AC, Buffa P, Castagnola E, Haupt R. Incidence of indwelling central venous catheter-related complications using the Sri Paran technique for device fixation in children with cancer. Pediatr Surg Int. 2009;25(7): 591-594.

45. Fratino G, Castagnola E, Carlini C, et al. A single institution observational study of early mechanical complications in central venous catheters (valved and open-ended) in children with cancer. Pediatr Surg Int. 2004;20(9):704-707.

46. Fratino G, Molinari AC, Mazzola C, et al. Prospective study of indwelling central venous catheter-related complications in children with broviac or clampless valved catheters. J Pediatr Hematol Oncol. 2002;24(8):657-661.

47. Jacobs BR, Schilling S, Doellman D, Hutchinson N, Rickey M, Nelson S. Central venous catheter occlusion: a prospective, controlled trial examining the impact of a positive-pressure valve device. JPEN J Parenter Enteral Nutr. 2004;28(2): 113-118.

48. Pittiruti M, Emoli A, Porta P, Marche B, DeAngelis R, Scoppettuolo G. A prospective, randomized comparison of three different types of valved and non-valved peripherally inserted central catheters. J Vasc Access. 2014, In press.

49. Crocoli A, Martucci C, Leopardi E, et al. A dedicated protocol and environment for central venous catheter removal in pediatric patients affected by onco-hematological diseases. J Vasc Access. 2014; Epub ahead of print.

50. Lee ACW. Elective removal of cuffed central venous catheters in children. Support Care Cancer. 2007;15(7):897-901.

51. Maizlin I, Carpentier H, Bliss D. Difficult extraction of long-term central venous catheters in children-case report. J Pediatr Surg. 2010;45(8):1720-1723.

52. Pittiruti M, Cina A, Cotroneo A, Di Stasi C, Malerba M, Cina G. Percutaneous intravascular retrieval of embolised fragments of long-term central venous catheters. J Vasc Access. 2000;1(1):23-27.

53. Guttmann DM, Trerotola So, Clark TW, et al. Malfunctioning and infected tunneled infusion catheters: over-the-wire catheter exchange versus catheter removal and replacement. J Vasc Interv Radiol. 2011;22(5):642-646, quiz 646. 